\title{
Percepción del paciente en la fase transoperatoria sobre el cuidado invisible de los profesionales de enfermería del Centro Quirúrgico en una clínica privada de Lima
}

Perception of patient in the intraoperative phase about the invisible care of nursing professionals in Surgical Center in a Private Clinic Of Lima

Poliana Salvanha Santos ${ }^{1 *}$, Keila Miranda Limachi²

\section{RESUMEN}

Objetivo: El estudio tuvo como objetivo determinar la percepción del paciente, en la fase transoperatoria, sobre el cuidado invisible de los profesionales de Enfermería del Centro Quirúrgico en una clínica privada de Lima. Metodología: Estudio de diseño cuantitativo y tipo observacional, descriptivo, prospectivo y transversal. Se estudió a 90 pacientes que fueron atendidas por profesionales de enfermería en un Centro Quirúrgico. En el análisis estadístico se utilizó frecuencias absolutas y relativas, promedio, desviación estándar y rangos. Resultados: Se encontró una edad media de 33,3 años, predominó el sexo femenino (83,3\%) y primera cirugía (47,2\%). La mayoría presentó un trato respetuoso favorable $(81,1 \%)$, percibieron alta tranquilidad $(81,1 \%)$, alta relación interpersonal (97,8\%), alta empatía (78,9\%), y el 95,6\% presentó una alta seguridad y privacidad. Conclusiones: La mayoría de pacientes percibió un cuidado invisible favorable, en la fase transoperatoria, de las profesionales de enfermería del Centro Quirúrgico de una clínica privada.

Palabras clave: Cuidado invisible, centro quirúrgico, percepción del paciente

\begin{abstract}
Objective: The aim of the study was to determine the patient's perception in the intraoperative phase about the invisible care of nursing professionals of Surgery Center in a private clinic in Lima. Methods: Design study was quantitative and type research was observational, descriptive, prospective and transversal. The study included 90 patients who were treated by nurses in a surgical center. Absolute and relative frequencies, mean, standard deviation and ranges were used in the statistical analysis. Results: It was found a mean age of 33.3 years, $83.3 \%$ were female and $47.2 \%$ said to be its first surgery. The $81.1 \%$ of patients had a favorable respectful treatment, $81.1 \%$ perceived a high tranquility, $97.8 \%$ perceived high interpersonal relationship, 78.9\% perceived high empathy and $95.6 \%$ he presented a high security and privacy. Conclusions: In conclusion, most patients perceived a favorable invisible care in the intraoperative phase, by the nurses of the Surgical Center in 2016 Good Hope Clinic.
\end{abstract}

Keywords: Invisible care; surgical center, the patient's perception. 


\section{INTRODUCCIÓN}

La enfermería promueve y mantiene la salud a través de acciones de cuidado, auxiliando a las personas a superar los efectos de la enfermedad (Gutiérrez y Hernández, 2006). Mucho de los cuidados de enfermería son cuidados que se pueden ver y medir, sin embargo, hay una clase de cuidados que no se pueden registrar, estos son los cuidados invisibles. Aunque existen algunos datos favorables del cuidado invisible del personal de enfermería, esta información no es suficiente para llevarla a la práctica, por lo cual se debe considerar el cuidado humanizado de manera integral como parte de la calidad de su labor profesional. Muchos son los estudios que hablan de la percepción del cuidado del paciente quirúrgico en el preoperatorio, pero poco se ha encontrado durante el transoperatorio. En este contexto, se plantean las siguientes inquietudes: $\mathrm{Al}$ ingresar al centro quirúrgico y al momento de la propia cirugía, cuando los pacientes están conscientes, ¿cómo percibe el paciente el cuidado de la enfermera que lo recibe dentro de sala? ¿Se siente único y respetado? ¿Perciben a las enfermeras como seres amables y empáticas? ¿Siente que su privacidad y seguridad son preservadas o se siente que el entorno es más importante que su propia presencia en la sala?

Es bajo estas circunstancias que se delimita el problema de la investigación, por ello el objetivo de la presente investigación es determinar la percepción del paciente en la fase transoperatoria sobre el cuidado invisible de los profesionales de Enfermería del Centro Quirúrgico en una clínica privada de Lima 2016. Esta investigación permitirá identificar y sistematizar la información obtenida sobre el cuidado invisible, asimismo, aportará conocimiento actualizado para futuras investigaciones.

Además, los resultados obtenidos brindarán conocimientos y dará una visión que permitirá identificar los aspectos positivos, negativos y las falencias que están ocurriendo en el quehacer diario de la profesión, en cuanto al cuidado humanizado e invisible.

\section{MATERIAL Y MÉTODOS}

El estudio es observacional, descriptivo y transversal. La población está representada por pacientes intervenidos en el quirófano de la clínica Good Hope y que están despiertos durante el acto quirúrgico. Según las estadísticas, en el centro quirúrgico se atiende un promedio de 253 pacientes mensualmente. El tamaño de la muestra fue calculado mediante la fórmula de población finita, por antecedentes (Gonzáles, 2013) se asumió una frecuencia favorable de cuidado invisible del 30\% y un error de precisión del 7,6\%, resultando un tamaño de muestra de 90 pacientes. La técnica de recolección fue la encuesta. Para el análisis descriptivo se usó frecuencias relativas y frecuencias absolutas para variables cualitativas, para expresar las variables cuantitativas se usaron las medidas de tendencia central y de dispersión. Las respuestas de cada reactivo positivo fueron asignadas de la siguiente forma. Nunca (0), A veces (1) y Siempre (2), mientras que los valores se invirtieron en las preguntas de forma negativa. Los valores finales del cuidado invisible fueron clasificados en función a los percentiles de la siguiente forma: Desfavorable $(<\mathrm{P} 50)$, Medianamente favorable (P50 - P80), Favorable $(>\mathrm{P} 80) . \mathrm{f}$

\section{RESULTADOS}

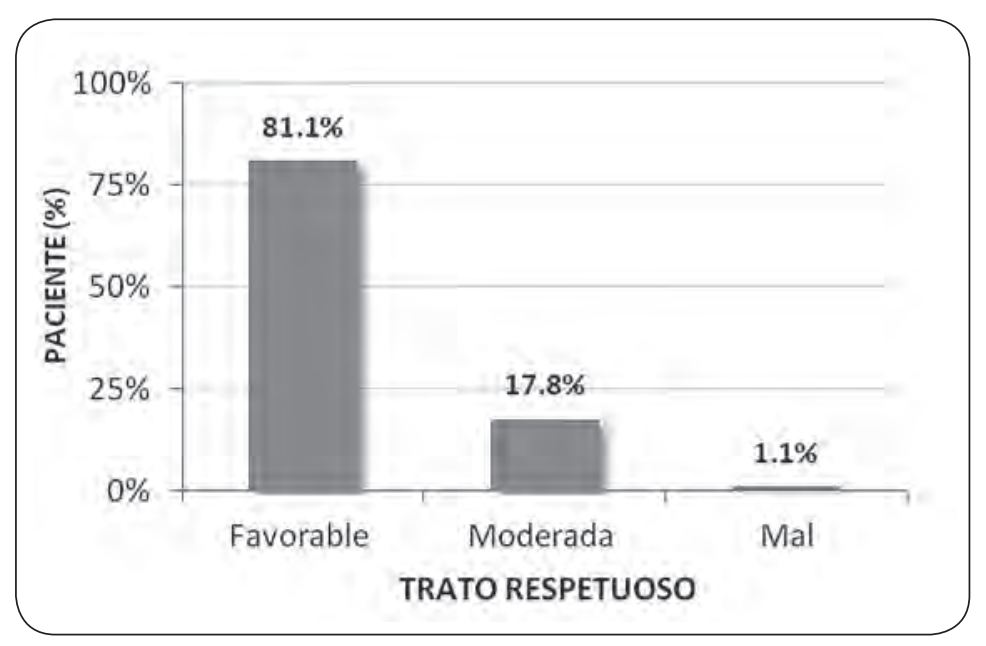

Figura 1. Percepción por el paciente según la dimensión Trato Respetuoso 
En la Figura 1 la mayoría de pacientes tuvieron una percepción favorable sobre la dimensión trato respetuoso del cuidado invisible de los profesionales de enfermería que laboran en Centro Quirúrgico (81,1\%).

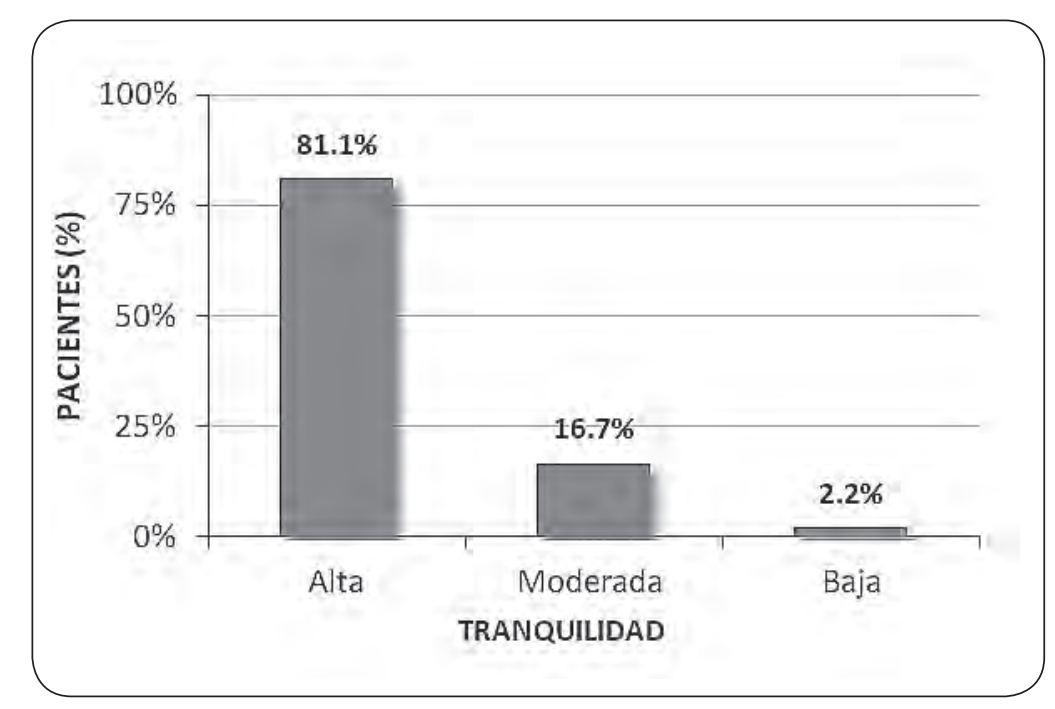

Figura 2. Percepción por el paciente según la dimensión Tranquilidad

La percepción de la mayoría de pacientes según la dimensión tranquilidad fue favorable $(81,1 \%)$, mientras el $16,7 \%$ percibió una moderada tranquilidad y dos pacientes percibieron un nivel bajo de tranquilidad. Los ítems "siempre la enfermera usa un tono de voz suave y toma unos minutos para hablar con el paciente” fueron las más frecuentes.

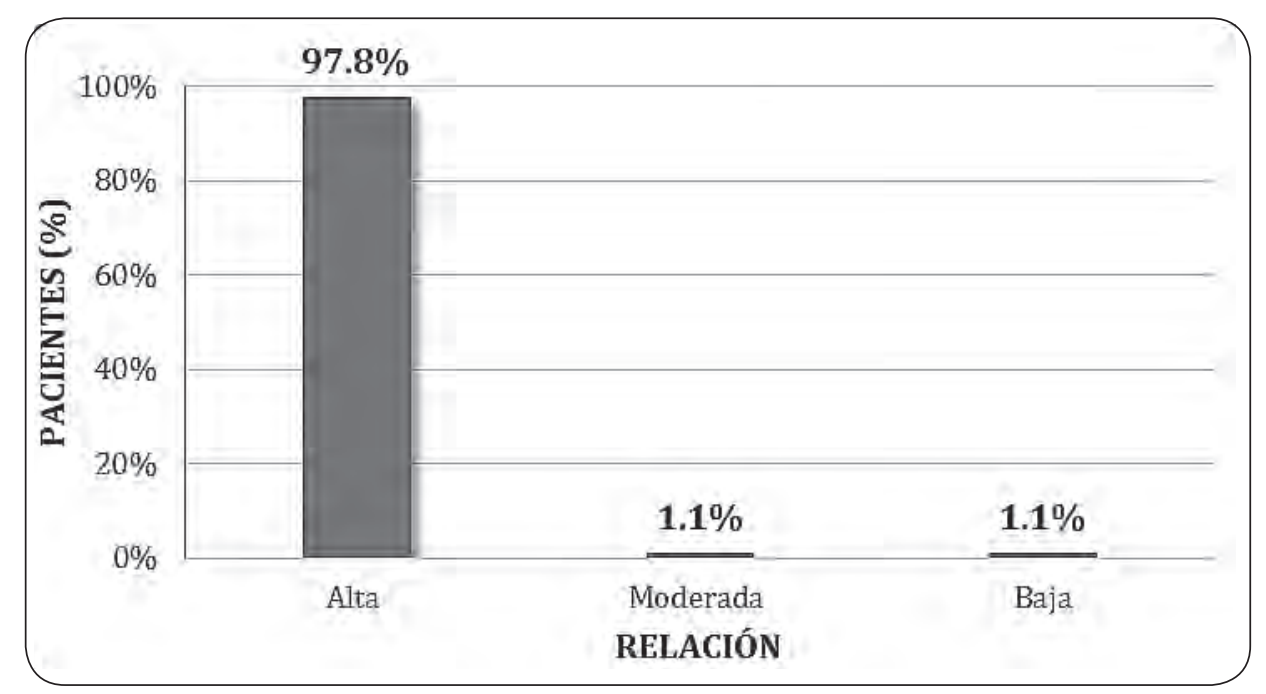

Figura 3. Percepción por el paciente según la dimensión Relación

Según la percepción del paciente en la dimensión Relación, del 100\% (90), el 97,8\% de los pacientes perciben una alta relación, el 1,1\%(1) percibe una moderada y baja relación respectivamente. Los ítems de la categoría alta se relacionan cuando el paciente refiere que la enfermera ha sido amable en su ingreso en el Centro Quirúrgico, paciente al hablar y ser delicada al momento de cuidarlo. 
Percepción del paciente en la fase transoperatoria sobre el cuidado invisible de los profesionales de enfermería del Centro Quirúrgico en una clínica privada de Lima

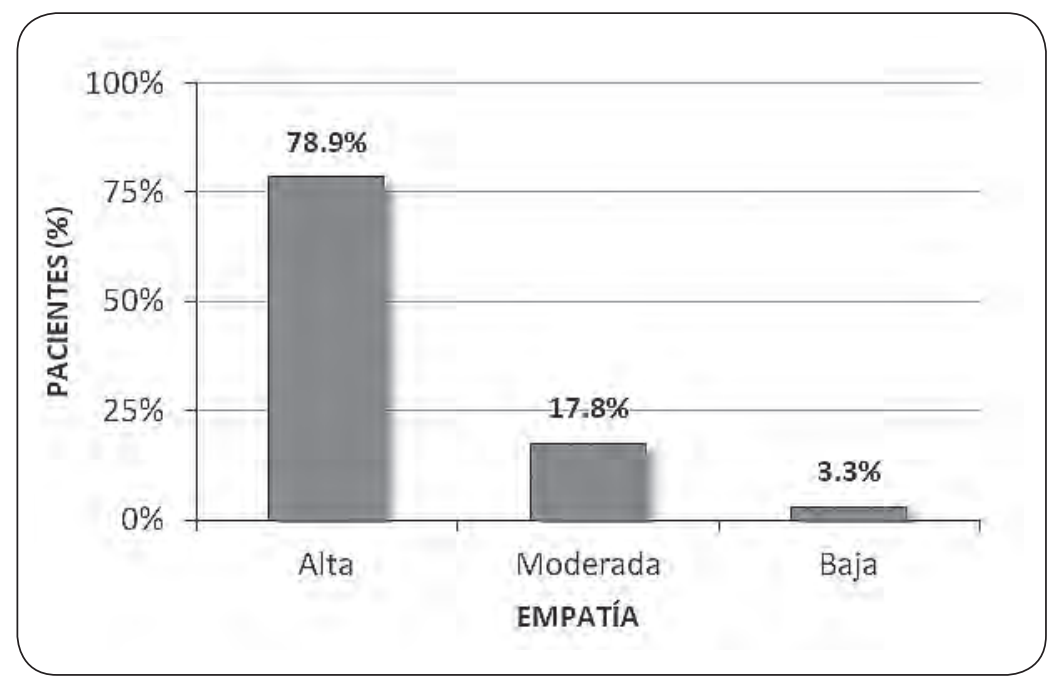

Figura 4. Percepción por el paciente según la dimensión Empatía

En cuanto a la percepción del paciente en la dimensión Empatía, el 78,9\% (71) pacientes perciben una alta empatía, el 17,8\%(16) pacientes perciben una moderada empatía y el 3,3\% (3) pacientes perciben una baja empatía. Además los ítems de la categoría alta se relacionan cuando el paciente manifiesta que siempre la enfermera se muestra atenta, es solidaria, está pendiente con sus necesidades, comprende sus dudas y ofrece su ayuda rápidamente.

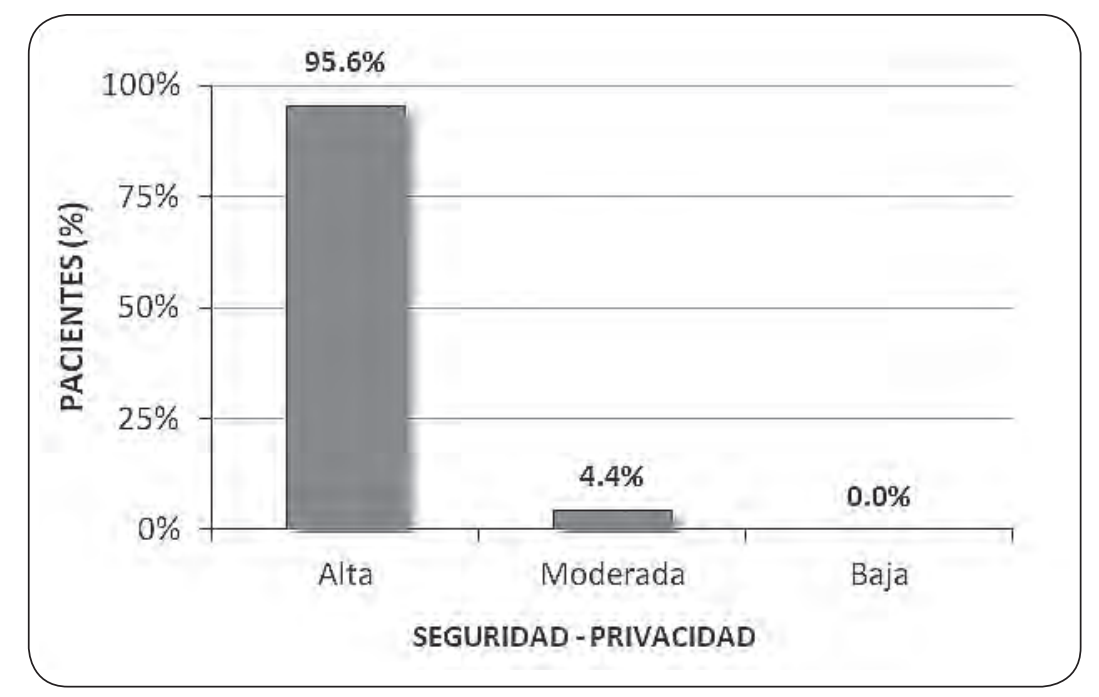

Figura 5. Percepción percibida por el paciente según la dimensión Seguridad-Privacidad

Respecto a la percepción del paciente en la dimensión seguridad-privacidad, el 95,6\% (86) pacientes perciben una alta seguridad-privacidad, donde los ítems de la categoría alta se relacionan cuando el paciente manifiesta que siempre la enfermera lo trata con respeto, sentirse seguro en la camilla donde lo traslada a la sala de operaciones, recibir auxilio para trasladarse de la cama quirúrgica a la camilla, y que la enfermera cuide su intimidad durante la preparación para la cirugía. 
Tabla 1

Percepción percibida por el paciente sobre el cuidado de los profesionales de Enfermería en la clínica privada de Lima 2016

\begin{tabular}{lcc}
\hline Percepción & $\mathrm{N}$ & $\%$ \\
\hline Favorable & 88 & $97,8 \%$ \\
Medianamente favorable & 1 & $1,1 \%$ \\
Desfavorable & 1 & $1,1 \%$ \\
Total & 90 & $100,0 \%$ \\
\hline
\end{tabular}

Según el consolidado de las 5 dimensiones estudiadas, en la presente investigación, se puede observar que la percepción de los pacientes en la fase transoperatoria sobre el cuidado invisible de los profesionales de enfermería que labora en Centro Quirúrgico fue favorable en un $97,8 \%$, una percepción medianamente favorable 1,1\% y desfavorable también un 1,1\%.

\section{DISCUSIÓN}

De acuerdo a la investigación, se evidenció que la edad promedio de los pacientes fue 33,3 años, donde el 88,9\% se encontraban entre 20 y 40 años, asimismo, el $83,3 \%$ de los pacientes son de sexo femenino y la gran mayoría tiene estudios superiores (98,9\%). Por su parte, Carrasco (2008) encontró que el 56,0\% de los pacientes tenían una edad menor a 60 años, además la mayoría tuvo grado de instrucción superior $(56,0 \%)$. Sin embargo, difieren en el género y en el número de cirugías, donde sostiene que el 58,0\% de los pacientes son de sexo masculino y refieren que han tenido más de tres cirugías (40,0\%).

Respecto a la dimensión trato respetuoso, se encontró que el $81,1 \%$ percibe favorablemente, $17,8 \%$ percibe moderadamente y el $1,1 \%(1)$ percibe un mal trato; resultado similar a lo encontrado en el estudio de Gonzales (2013), donde tuvo como objetivo determinar la percepción que tiene el paciente sometido a cirugía oftalmológica acerca del cuidado que brinda la enfermera en el servicio de Cirugía de día, encontrando que el $72,4 \%$ tiene una percepción medianamente favorable, 15.4\% favorable y 12,2\% desfavorable. La necesidad que presenta el paciente es una sensación de carencia que debe ser satisfecha por el profesional, debido que ha de confiar en este como una persona que le brindará el mejor cuidado; para ello, el profesional de enfermería debe conocer y comprender la conducta del paciente en un medio extraño para él, y por lo anterior debe compartir experiencias humanas mediante la relación de respeto entre el profesional y el paciente (Silva, Ramón, Vergaray, Palacios y Partenazi, 2015).
La percepción del usuario acerca del trato digno por Enfermería fue adecuada en 91\%. Además, resultó importante para el usuario que la enfermera le ofrezca condiciones que guarden su intimidad y pudor, como lo comprobó Ortiz (2014) al determinar que la satisfacción de los usuarios se relaciona con el confort y la limpieza de las instalaciones; al respecto, Bautista (2008) plantea que la relación enfermerapaciente adquiere mayor relevancia, si el personal se interesa por brindarle comodidad, privacidad y un ambiente agradable al usuario. Los pacientes no solo perciben los elementos racionales en el cumplimiento de sus derechos, sino que asignan componentes emocionales, esto quiere decir que el valor agregado del trato al paciente está en respetar sus emociones, particularmente cuando se trata de su estado de salud.

Por otra parte, en la dimensión tranquilidad, se encontró que la percepción del paciente fue alta mostrando un $81,1 \%$, asimismo, el $16,7 \%$ de los pacientes perciben una moderada transmisión de tranquilidad y finalmente el 2,2\% percibe una baja transmisión de tranquilidad; resultado que no varía mucho por Gonzáles (2013) donde el 50\% de los pacientes tienen una percepción medianamente favorable, el 35\% se encuentra favorable y el $15 \%$ se encuentra desfavorable. Brindar tranquilad al paciente incluye saber qué decir y la forma cómo decirlo; incluso involucra todo aquello que no se dice o lo que se denomina comunicación no verbal; a pesar de los buenos porcentajes encontrados es necesario que los profesionales de enfermería sigan fortaleciendo sus habilidades psicoafectivas para actuar en concordancia con el paciente respecto a su cuidado; y poder ofrecer el estado de calma que este necesita. La comunicación con los pacientes no solo está dada por palabras, pues como expresa De La Cuesta (2004) “el desafío para los cuidadores ahora es hacerse entender del paciente y entenderle a él, hablar con él en un lenguaje que para ambos tenga sentido, y cuando no haya palabras, encontrar la manera de comunicarse”.

En relacion a la dimensión relación, el 97,8\% de los pacientes perciben una alta relación con los profesionales de enfermería; resultados que son casi similares por Gonzáles (2013) donde el 60\% de los pacientes tienen una percepción medianamente favorable, el $30 \%$ de los pacientes se encuentran favorables y $10 \%$ de los pacientes están desfavorables. Por su parte, la investigación de Bernal (2011) en la evaluación del trato digno, con respecto a la relación enfermera-paciente, el 89\% de las mujeres consideraron que esta relación fue buena, 9\% que fue regular y el $2 \%$ que fue mala. El desarrollo de una relación enfermera-paciente de forma adecuada permite valorar de forma exhaustiva el estado de salud del paciente e identificar rápidamente sus 
necesidades; por ello se le considera el punto clave de los cuidados de enfermería. El trato digno incluye el saludo amable hacia el paciente, la presentación del personal de salud, hablar al paciente por su nombre, la explicación de actividades o procedimientos que se van a realizar, salvaguardar la intimidad del paciente, el hacer sentir seguro al paciente, ser tratado con respeto, brindar información acerca de los cuidados posteriores al procedimiento, no solo al paciente sino al familiar responsable y, por último, la satisfacción del paciente con respecto al trato del personal que le atendió.

En cuanto a la dimensión empatía, el 78,9\% (71) pacientes perciben una alta empatía, el 17,8\%(16) perciben una moderada empatía y el 3,3\% (3) perciben una baja empatía, mostrando que siempre la enfermera se muestra atenta, es solidaria, está pendiente con sus necesidades, comprende sus dudas y ofrece su ayuda rápidamente; resultados congruentes por Gonzales (2013) donde el 57\% de los pacientes presentan una percepción favorable, mostrando que el profesional de enfermería siempre comprende sus dudas. En cambio, difiere con Carrasco (2008) el cual encontró que el $38 \%$ de los pacientes manifiestan una opinión desfavorable, donde los ítems concernientes fueron la enfermera muestra fastidio y desinterés durante la atención, no brinda tranquilidad y seguridad, además, no satisface las necesidades y requerimientos del paciente.

Sobre la dimensión seguridad-privacidad, el 95,6\% (86) de los pacientes perciben una alta seguridadprivacidad. Resultado que concuerda con lo reportado por Villajuan (2010) quien encontró que el 71,4\% de los pacientes percibieron un nivel alto de seguridad y 25,3\% su percepción fue de nivel medio. Otros autores como Gonzales y Quintero (2009) encontraron que el $78 \%$ siempre percibió la priorización al ser cuidado, 8\% casi siempre, 9\% algunas veces y 5\% nunca percibió la priorización del cuidado, donde evaluaron el respeto, le llaman por su nombre, respetan sus decisiones, respetan la intimidad y ante cualquier eventualidad lo ponen en primer lugar.

La percepción de los pacientes intervenidos en el quirófano acerca del cuidado que brinda los profesionales de enfermería en la clínica Good Hope, del $100 \%$, es decir de los 90 pacientes, el 97,8\% (88) perciben favorablemente la atención de los profesionales de enfermería, 1,1\% (1) percibe medianamente favorable y $1,1 \%$ (1) percibe una desfavorable atención de los profesionales de enfermería; hallazgos que son congruentes por lo reportado en Villajuan (2010) quien encontró que el $60,7 \%$ de los pacientes presentaron un alto nivel de satisfacción con el trato brindado. Asimismo, para González y Quintero (2009) encontraron que el 73\% de las mujeres hospitalizadas percibieron siempre el cuidado humanizado del personal de enfermería, el $11 \%$ casi siempre percibió el cuidado humanizado, el $12 \%$ algunas veces percibió el cuidado humanizado y el 4\% nunca percibió el cuidado humanizado por parte del personal de enfermería. Finalmente, se hacen cada vez más necesarios los profesionales de enfermería que estén preparados para desarrollar sus funciones de cuidado invisible del paciente quirúrgico; con ello mejorará la calidad en la atención del paciente, siendo así más holística y humanizada.

\section{Declaración de financiamiento y de conflicto de intereses:}

El estudio fue financiado por los autores, quienes declaran no tener algún tipo de conflicto de interés en la investigación realizada.

\section{Correspondencia:}

Poliana Salvanha Santos

Clínica Good Hope. Malecón Balta 956, Miraflores, Lima Perú.

e-mail: polisalvanha@hotmail.com.

\section{REFERENCIAS BIBLIOGRÁFICAS}

Bautista R. (2008). Percepción de la calidad del cuidado de enfermería en la Escuela Superior de Enfermería Francisco de Paula Santander. Rev. Aquichan, 8 (1): 74-84.

Bernal M, Ramírez A, Ramírez G. (2011). Percepción de trato digno de usuarias que acuden al servicio de interrupción legal del embarazo (ILE). Rev. Enferm. Univ. ENEOUNAM, 8 (3): 21-27.

Carrasco, L. (2008). Opinión que tiene el paciente quirúrgico ambulatorio sobre la atención humanizada que brinda el profesional de enfermería en la sala de operaciones del Hospital Alberto Sabogal Sologuren- EsSalud. (Tesis de grado). Universidad Nacional Mayor de San Marcos. Lima. pp. 1-104.

De la Cuesta, C. (2004). La artesanía del cuidado: Estrategias para cuidar pacientes con demencia avanzada. En: Cuidado artesanal la invención ante la adversidad. 3 ed. Medellín: Universidad de Antioquía. p.115.

Gonzáles, M. (2013). Percepción del paciente sometido a cirugía oftalmológica acerca del cuidado que brinda la enfermera en el servicio de cirugía de día en el Hospital 
Nacional Guillermo Almenara Irigoyen Lima-Perú 2013. (Tesis de especialidad). Universidad Nacional Mayor de San Marcos. Lima. pp. 1-56

González, J., y Quintero, D. (2009). Percepción del cuidado humanizado en pacientes que ingresan al servicio de hospitalización de urgencias de ginecoobstetricia de una institución de II y III nivel de atención. (Tesis de grado). Pontificia Universidad Javeriana. Bogotá. pp. 1-101

Gutiérrez, L., y Hernández, S. (2006). Significado del discurso de cuidado del docente en la formación del estudiante frente al acto de cuidado humanizado. Biblioteca Las casas; 3 (1):1-97.
Ortiz, R. (2004). Satisfacción de los usuarios de 15 hospitales de Hidalgo, México. Rev Esp Salud Pública, 78 (4): 527537.

Silva J, Ramón S, Vergaray S, Palacios V, Partezani R. (2015). Percepción del paciente hospitalizado respecto a la atención de enfermería en un hospital público. Rev. Enfermería Universitaria, 12 (2): 80-87.

Villajuan, E. J. (2010). Nivel de satisfacción del paciente acerca del cuidado enfemero que recibe en el Servicio de Cirugía de Día del HNGAI. (Tesis de especialidad). Universidad Nacional Mayor de San Marcos. Lima. pp. $1-50$. 